\title{
Engagement in Different Sport Disciplines During University Years and Risk of Locomotive Syndrome in Older Age: J-Fit ${ }^{+}$Study
}

\section{Shaoshuai Shen}

Juntendo University

Koya Suzuki ( $\square$ koyafitness@gmail.com )

Juntendo University https://orcid.org/0000-0002-2089-0886

Yoshimitsu Kohmura

Juntendo University

Noriyuki Fuku

Juntendo University

Yuki Someya

Juntendo University

Hisashi Naito

Juntendo University

Research article

Keywords: university sports club, sport disciplines, locomotive syndrome, Japanese men, historical cohort, university athletes, middle-aged men, older men

Posted Date: November 20th, 2020

DOI: https://doi.org/10.21203/rs.3.rs-107341/v1

License: (c) (i) This work is licensed under a Creative Commons Attribution 4.0 International License. Read Full License 


\section{Abstract}

Background: Among former Olympic-level athletes, engagement in different sport disciplines is associated with reduced mortality risk in subsequent years. However, limited evidence is available on whether engagement in different sport disciplines at a young age is associated with locomotive syndrome (LS) risk later in life. This study examined the relationship between engagement in different sport disciplines during university years and LS risk in older age among former university athletes.

Methods: Participants were 274 middle-aged and 294 older men alumni who graduated from a school of physical education in Japan. Data on university sports participation and club membership as well as LS risk were collected using questionnaires. University clubs were classified into three groups of cardiovascular intensity (low, moderate, high), following the classification system of sport disciplines by the American College of Cardiology. This classification considers the static and dynamic component of an activity, which correspond to the estimated percent of maximal voluntary contraction reached and to that of maximal oxygen uptake achieved, respectively. University clubs were grouped based on the risk of bodily collision (no, yes) and extent of physical contact (low, moderate, high). Relationships between engagement in different sport disciplines and LS risk were analyzed using Cox proportional hazards models, adjusted for relative risks.

Results: Adjusted hazard ratios and $95 \%$ confidence intervals associated with the low, moderate, and high cardiovascular intensity sports were 1.00 (reference), $0.48(0.22-1.06, \mathrm{P}=0.07)$, and $0.44(0.20-0.97, \mathrm{P}=$ 0.04 ) in older men, respectively; however, there was no significant association between these parameters among middle-aged men. Engagement in sports associated with physical contact and collision did not affect LS risk in either group.

Conclusions: Engagement in sports associated with high cardiovascular intensity during university years may reduce the risk of LS in later life. Encouraging young people to participate in such activities might help reduce LS prevalence among older populations.

\section{Background}

As the average age of the Japanese population increases, the number of older adults in need of nursing care grows annually, driving long-term care insurance expenditure, which more than doubled from 4 to 10 trillion yen between 2000 and 2018 [1]. In this context, in 2007, the Japanese Orthopaedic Association introduced a concept of locomotive syndrome (LS) $[2,3]$ to describe people at high risk of musculoskeletal ambulation disability (associated with impaired mobility function such as sit-to-stand or gait) caused by diseases of the locomotor organ [4]. This concept aims to raise awareness among people affected by or at risk of LS to maintain their health and delay or prevent the need for long-term care [5].

In Japan, LS has been identified as an independent risk factor for falls and reduced mobility involved in activities of daily living [6, 7], causing older adults to require long-term care [8]. In fact, LS-related complications, such as fractures, falls are the fourth, and musculoskeletal disorders are the fifth leading 
cause of need for long-term care [9]. Meanwhile, although LS is a risk factor for older adults, its prevalence among men and women aged $<40$ years is estimated at $13 \%$ and $19.4 \%$, respectively [6]. LS among relatively young people is a cause for concern as the syndrome tends to persist into older age, driving future long-term need for care. To develop an effective approach to prevention, it is necessary to understand what activities, including types of sport, performed at a young age can reduce the risk of LS.

Health benefits of physical activity and exercise are well known; governments and specialist societies worldwide recommend that people regularly participate in physical exercise [10-12]. A growing body of evidence demonstrates the benefits of physical activity among school-aged children and adolescents [13]. For example, Kidokoro et al. reported that moderate to vigorous physical activity in this age group can reduce the adverse effects of television viewing on cardiorespiratory fitness [14]. Moreover, other studies suggest that higher levels of physical activity are associated with lower risk of cardiovascular disease [15], while endurance sports and vigorous activity can protect against coronary heart disease [16]. Furthermore, evidence suggests that increasing the level of physical exercise helps prevent type 2 diabetes mellitus [17]. Overall, physical exercise appears essential to health promotion and prevention of many diseases.

Nevertheless, longitudinal studies that examined the association between exercise intensity and longevity achieved conflicting results $[18,19]$. For example, Lee et al. demonstrated that total energy expenditure and energy expenditure from vigorous activities were inversely associated with mortality risk among Harvard alumni [18]; meanwhile, Zwiers et al. reported that engagement in high-intensity sports did not bring any survival benefits compared with low-intensity activities among former Olympic athletes [19]. These discrepancies might be due to between-study differences in target populations or definitions of "intensity"; as a result, interpretation of these findings remains controversial and whether high-intensity exercise protects against LS is subject to debate. Evidence on the association between engagement in different sports during university years and LS risk is scarce. However, understanding the impact of different intensity exercise performed during university years on LS risk in middle and older age is crucial to disease prevention policy and education.

This study examined the effects of engagement in different sports disciplines during university years on LS risk in middle and older age among school of physical education alumni. We hypothesized that highintensity exercise is associated with lower risk of LS in these groups.

\section{Methods}

\section{Study design and population}

The $\mathrm{J}-\mathrm{Fit}^{\natural}$ Study is a historical cohort study that investigates the association between physical fitness, motor ability, sports experience, sports club membership at a young age, and future diseases among alumni of physical education university. J-Fit ${ }^{\natural}$ Study details and participants' characteristic have been described previously $[20,21]$. 
This study included 1385 Japanese alumni of physical education university who had participated in a follow-up health examination and responded to a self-administered questionnaire at least once during 2007-2009, and 2011 [22]. In 2017, among 1385 alumni who had participated in the second follow-up health examination, $49.3 \%(n=683)$ of individuals consented to be contacted by us and were sent a questionnaire regarding their height, weight, body mass index (BMI), university sports club membership, recent habitual exercise, joint disease, smoking status, drinking status, and locomotive organ health (Loco-check) by post. Alumni who failed to respond $(n=702)$ or to provide information on their LS risk ( $n$ $=19)$ or sports club membership $(n=96)$ were excluded. Finally, 568 individuals were included in the analysis; the sample was dichotomized by age $<65(n=274)$ and $\geq 65(n=294)$ years (Fig. 1). Ethical approval was provided by the Faculty of Health and Sports Science, Juntendo University (No. 31 - 25).

\section{Classification of university sports club disciplines}

The university sports club membership was assessed with the following open-ended questions: "Did you belong to a university sports club while at university? What was its name? How many weekly practice sessions did you participate in? How many hours per session?" We classified sports club membership based on the American College of Cardiology's sports classification system [23]. This system defines three levels of cardiovascular intensity (low, moderate, high) based on the extent of dynamic or static exertion required to perform that sport during competition; these components correspond to the estimated percent of maximal oxygen uptake achieved and to that of maximal voluntary contraction reached, respectively. In addition, total cardiovascular intensity (low, moderate, high) is the sum of static and dynamic component values. The total cardiovascular demand is shown in green (low), orange (moderate), and red (high) in Fig. 2. This classification system also differentiates between sports that are associated with a risk of bodily collision, either with another player or with an object, projectile, or the ground; as well as the degree of risk to the athlete or others if a sudden syncopal event occurs. Based on these factors, we divided sports clubs into those associated with risk of bodily collision (including the danger of bodily collision and increased risk of syncope) and those without such risk [19]. Finally, we considered the American Academy of Pediatrics' classification of contact sports, which accounts for the risk of injury [24]. Sports clubs were categorized into non-contact (low), limited-contact (moderate), or high-contact (high) based on the relative risk of an acute injury to the athlete.

\section{Locomotive syndrome risk test}

The following seven items were included in the Loco-check questionnaire developed by the Japanese Orthopaedic Association (JOA) to evaluate locomotive organs: 1. You can't put on your sock standing on one leg; 2 . You often trip or slip around the house; 3 . You need to hold on to the handrail when climbing the stairs; 4 . You have difficulty doing moderately heavy housework; 5 . You have difficulty carrying home $2 \mathrm{~kg}$ of shopping (e.g., equivalent to two 1-L cartons of milk); 6. You can't walk for a quarter of an hour nonstop; 7. You can't make it across the road before the light turns red. Participants responded to these with either 'yes' or 'no'. Participants who answered 'yes' to one or more of these items, they were defined as subjects suspected of LS risk; participants who answered "no" to all seven items were defined as "no risk" of LS [25]. 


\section{Physical fitness and motor ability tests}

Since 1973, the study site has performed an annual test of physical fitness and motor ability of its students. Side-step test, vertical jump, back muscle strength, grip strength, trunk lift, trunk-forward flexion, and step-test are used to assess physical fitness. In addition, 50-meter run, and 1500-meter run, running long jump, hand-ball throw, and pull-up are used as motor ability tests. Details of these tests are presented in the Additional file 1. Tests results obtained in the fourth year of university by participating alumni were used in this study.

\section{Statistical analysis}

Statistical analyses were performed with IBM SPSS Statistics for Windows, version 21.0 (SPSS Inc., Chicago, IL, USA), and the level of significance was set at $p$-value $<0.05$. Continuous variables were expressed as medians (interquartile range). Categorical variables were presented as numbers (percentage). Cox proportional hazards model was used to examine the association between sports club membership during university and LS risk in later life. The following models were constructed in Cox proportional hazards analysis. Univariate analysis was adjusted for age, height, weight, joint disease, habitual exercise, and smoking and drinking status. Multivariate analysis was additionally adjusted for cardiovascular intensity, bodily collision, and physical contact. Independent sample t-test was used to examine differences in physical fitness and motor ability test results obtained at university by alumni included in the low vs. high total cardiovascular intensity group.

\section{Results}

Overall, 568 participants were included in this study, including 274 middle-aged and 294 older men.Table 1 summarizes the characteristics of the study population in all participants and of middleaged men and older men. Among middle-aged men, during the follow-up period, LS risk was suspected in 6 (13.6\%), 23 (13.8\%), and 11 (17.5\%) participants of total cardiovascular intensity (low, moderate, high), respectively (Table 1). In older men, during the follow-up period, LS risk was suspected in 31 (53.4\%), 62 (34.6\%), and 21 (36.8\%) participants of total cardiovascular intensity (low, moderate, high), respectively (Table 1).

Table 2 demonstrates the university sports club disciplines stratified for three levels of total cardiovascular intensity (low, moderate, and high). The number of volleyball club members in the low cardiovascular intensity exercise group was 61 (59.8\%). The number of gymnastics club members in the moderate cardiovascular intensity exercise group was 65 (18.8\%). The number of basketball club members in high cardiovascular intensity exercise group was 61 (50.8\%) (Table 2).

In univariate models, there was no association between any sports category and the risk of LS in either middle-aged or older men (Table 3). In multivariate analysis, neither risk of bodily collision nor physical contact were associated with the risk of LS in either middle-aged or older men. However, in multivariate analysis, engagement in exercise of increased cardiovascular intensity was associated with lower risk of 
LS; hazard ratio for moderate-intensity exercise was 0.48 (95\% confidence interval [Cl]: 0.221-1.062; $\mathrm{P}=$ $0.070)$, and that for high-intensity exercise was 0.44 ( $95 \% \mathrm{Cl}$ : $0.200-0.969 ; \mathrm{P}=0.042)$ among older men.

Finally, there were differences between low and high cardiovascular intensity exercise groups in results of 1,500-m run and running long jump $(P<0.05)$ (Table 4).

\section{Discussion}

The present study has shown that engagement in low to moderate cardiovascular intensity sports during university years was not associated with the risk of LS in later life. Similarly, engaging in sports with risk of collision or bodily contact during university did not impact LS risk in older age. In contrast, engagement in high cardiovascular intensity sports during the same period was associated with significantly lower risk of LS among older men. These findings suggest that engaging in sports of high cardiovascular intensity at younger age may help reduce the risk of LS in later adulthood.

In the present study, men aged $\geq 65$ years were more likely to be affected by LS (low: $53.4 \%$, moderate: $34.6 \%$, high: $36.8 \%$ ) than were men aged < 65 years (low: $13.6 \%$, moderate: $13.8 \%$, high: $17.5 \%$ ); these estimates are consistent with those previously reported [26]. Concurrently, this study suggests that engagement in high-intensity sports during university does not reduce the risk of LS in middle-aged men compared to engagement in low-intensity sports. Yoshimi et al. [27] reported that the total body muscle mass decreases in Japanese men around the age of 40 years and then at the age of 65 years and beyond, where it tends to decrease rapidly. Kortebein et al. [28] reported that older people tend to lose lean leg mass faster than young people. In addition, Goodpaster et al. [29] reported that age-associated loss of muscle mass is a major factor in age-associated muscle strength decline. Altogether, these findings suggest that decline in physical strength is slower among middle-aged than among older adults. Compared with older adults, middle-aged adults tend to maintain relatively high levels of physical function. This discrepancy might account for the differences in impact of high-intensity exercise during university years on the risk of LS in older age, which, in contrast to older adults, was not observed among middle-aged men. In this study, older men with history of high-intensity exercise during university years were at lower risk of LS than their counterparts without such history.

Engagement in high cardiovascular intensity sports during university years reduced the risk of LS in older men. Specifically, playing basketball or team handball, and practicing middle-distance running, among others, were associated with lower LS risk compared to playing volleyball, baseball, or table tennis. These results are similar to those from previous studies. For example, Lemez and Baker [30] reported that, compared with the general population, elite athletes had longer life expectancy. In addition, Sarna et al. [31] reported that among world-class male athletes, those engaged in endurance and team sports had lower premature mortality rates. Teramoto and Bungum [32] speculate about the reasons for these findings; they propose that participating in higher volumes of exercise training will lead to higher physical fitness levels, and the possibility that elite athletes are composed of the healthiest and healthiest individuals. Our findings on the differences between low and high cardiovascular intensity exercise 
groups in physical fitness and motor ability tests results during university years support this interpretation; specifically, participants engaged in high-intensity sports performed better on 1,500 m run and running long jump tests than those engaged in low-intensity sports. These findings suggest that people who are engaged in high cardiovascular intensity sports during university years have higher physical fitness levels than those who are engaged in low cardiovascular intensity sports. We speculate that this relatively higher physical fitness levels are maintained into older age, reducing the risk of LS.

Our findings are in contrast to those of some previous studies that showed no association between engagement in high cardiovascular intensity sports and premature mortality risk in later life among former Olympic athletes [19]. However, it is plausible that this kind of activity does not affect premature mortality risk while concurrently reducing the risk of LS.

Collision risk and physical contact associated with different university sports did not affect the risk of LS in later life in the present study. However, higher mortality risk has been associated with disciplines involving high levels of physical contact among former Olympic athletes [19], who experience a significant burden of injuries associated with their chosen disciplines [33, 34]. These injuries may gradually accumulate and have long-term effects [19]. This discrepancy in findings between the present and previous study might be accounted for as follows. The previous studies involved former Olympic athletes, whose training frequency and intensity was likely higher than that of the present study participants; as a result, the cumulative extent of sustained injuries as well as rate of physical impact was likely grater among the former than among the latter group. Although we have not found evidence that contact sports played during university years increase the risk of LS in later life, avoiding these sports reduces the risk of injuries and might thus be beneficial for overall health; using protective gear while playing these sports might also help prevent injury and protect health.

This study has several limitations, which should be considered when interpreting its findings. First, since the department of physical education of our university did not enroll female students before 1991, our survey included only men. Future studies should examine these associations in women. Second, this study involved alumni of a physical education department at a university; whether the presented findings generalize to a broader population is not clear. Third, we evaluated sports club membership during university years and LS risk in older age using a self-report questionnaire, which may lead to misclassification. Finally, our study did not consider changes to levels and type of sports practiced during the follow-up period. However, when examining the relationship between sports engagement during university years and the risk of LS in later life, we adjusted our estimates for present exercise habits.

\section{Conclusions}

We conducted a historical cohort survey to investigate the relationship between engagement in different sport disciplines during university and LS risk in later life. Our study demonstrated an association between engagement in high level of cardiovascular intensity sports during university years and the risk 
of LS in later life. Our findings suggest that engagement in high cardiovascular intensity sports during university years may reduce the risk of LS in later life.

\section{Abbreviations}

BMI

body mass index; Cl:Confidence interval; HR:hazard ratios; JOA:Japanese Orthopaedic Association; LS:locomotive syndrome

\section{Declarations}

\section{Ethics approval and consent to participate}

All subjects provided written informed consent for data collection and storage as part of this study. The study protocol was approved by the Ethics Committee of Juntendo University (Reference No. 31-25).

\section{Consent for publication}

Not applicable.

\section{Availability of data and materials}

The datasets analyzed in the current study are available from the corresponding authors on reasonable request.

\section{Competing interests}

The authors declare that they have no competing interests.

\section{Funding}

This study was supported by Grant-in-Aid for Scientific Research (KAKENHI) from the Japanese Society for the Promotion of Science (grant number 20 K19516), the Institute of Health and Sports Science \& Medicine, Juntendo University, and the MEXT-Supported Program for the Private University Research Branding Project. The funding body had no role in the study design, data collection and analysis, or preparation of the manuscript.

\section{Authors' contributions}

SS and KS conceived and designed the study, performed statistical analyses, and drafted the manuscript; $\mathrm{HN}$ conceptualized and oversaw the study, reviewed and revised the manuscript; KS, YK, NF, YS, and HN gave advice on methodology, and critically reviewed and revised the manuscript for important intellectual content. All authors contributed to writing, reviewing or revising the paper and read and approved the final 
manuscript. KS is the guarantor of this work, had full access to all the study data, and takes responsibility for its integrity and accuracy of data analysis.

\section{Acknowledgments}

We wish to thank all staff members who collaborated with us on data collection. We are also grateful to all alumni of Juntendo University and participants in this study.

\section{References}

1. Ikegami N. Financing Long-term Care: Lessons From Japan. Int J Health Policy Manag. 2019;8(8):462-466.

2. Nakamura K. The concept and treatment of locomotive syndrome: its acceptance and spread in Japan. J Orthop Sci. 2011;16(5):489-491.

3. Nakamura K, Ogata T. Locomotive Syndrome: Definition and Management. Clin Rev Bone Miner Metab. 2016;14:56-67.

4. Nakamura K. A "super-aged" society and the "locomotive syndrome". J Orthop Sci. 2008;13(1):1-2.

5. Ikemoto T, Arai YC. Locomotive syndrome: clinical perspectives. Clin Interv Aging. 2018;13:819-827.

6. Yoshimura N, Muraki S, Oka H, Tanaka S, Ogata T, Kawaguchi H, Akune T, Nakamura K. Association between new indices in the locomotive syndrome risk test and decline in mobility: third survey of the ROAD study. J Orthop Sci. 2015;20(5):896-905.

7. Yumura R, Ishibashi H, Fujita $\mathrm{H}$. Relationship between fall history and locomotion test and motor function measurements in community-dwelling middle-aged and elderly people (in Japanese). Rigaku-Ryouhou rinnsyou kenkyuu kyouiku (Physical Therapy-Clinical, Research, Education). 2016;23(1):40-46.

8. Japanese Orthopaedic Association. The Locomotive Challenge! website. 2020 (in Japanese). https://locomo-joa.jp/locomo/. Accessed 30 Mar 2020

9. Ministry of Health, Labour and Welfare. Comprehensive Survey of Living Conditions. 2016 (in Japanese). https://www.mhlw.go.jp/toukei/saikin/hw/k-tyosa/k-tyosa16/dl/16.pdf. Accessed 30 Mar 2020.

10. Haskell WL, Lee IM, Pate RR, Powell KE, Blair SN, Franklin BA, Macera CA, Heath GW, Thompson PD, Bauman A. Physical activity and public health: updated recommendation for adults from the American College of Sports Medicine and the American Heart Association. Circulation. 2007;116(9):1081-1093.

11. World Health Organization. Global recommendations on physical activity for health. 2010. https://www.who.int/dietphysicalactivity/global-PA-recs-2010.pdf. Accessed 28 Apr 2020.

12. Piercy KL, Troiano RP, Ballard RM, Carlson SA, Fulton JE, Galuska DA, George SM, Olson RD. The Physical Activity Guidelines for Americans. Jama. 2018;320(19):2020-2028. 
13. Janssen I, LeBlanc AG. Systematic review of the health benefits of physical activity and fitness in school-aged children and youth. International Journal of Behavioral Nutrition and Physical Activity. 2010;7(1):40.

14. Kidokoro T, Suzuki K, Naito H, Balasekaran G, Song JK, Park SY, Liou YM, Lu D, Poh BK, Kijboonchoo $\mathrm{K}$ et al. Moderate-to-vigorous physical activity attenuates the detrimental effects of television viewing on the cardiorespiratory fitness in Asian adolescents: the Asia-fit study. BMC Public Health. 2019;19(1):1737.

15. Kohl HW. Physical activity and cardiovascular disease: evidence for a dose response. Med Sci Sports Exerc. 2001;33(6):S472-S483.

16. Kujala UM, Sarna S, Kaprio J, Tikkanen HO, Koskenvuo M. Natural selection to sports, later physical activity habits, and coronary heart disease. British Journal of Sports Medicine. 2000;34(6):445-449.

17. Kelley DE, Goodpaster BH. Effects of exercise on glucose homeostasis in Type 2 diabetes mellitus. Med Sci Sports Exerc. 2001;33(6):S495-S501.

18. Lee I-M, Hsieh C-c, Paffenbarger RS, Jr. Exercise Intensity and Longevity in Men: The Harvard Alumni Health Study. Jama. 1995;273(15):1179-1184.

19. Zwiers R, Zantvoord FWA, Engelaer FM, van Bodegom D, van der Ouderaa FJG, Westendorp RGJ. Mortality in former Olympic athletes: retrospective cohort analysis. BMJ : British Medical Journal. 2012;345:e7456.

20. Someya Y, Kawai S, Kohmura Y, Aoki K, Daida H. Cardiorespiratory fitness and the incidence of type 2 diabetes: a cohort study of Japanese male athletes. BMC Public Health. 2014;14:493.

21. Kidokoro T, Kohmura Y, Fuku N, Someya Y, Suzuki K. Secular trends in the grip strength and body mass index of sport university students between 1973 and 2016: J-Fit+ study. Journal of Exercise Science \& Fitness. 2020;18(1):21-30.

22. Someya Y, Tamura Y, Kohmura Y, Aoki K, Kawai S, Daida H. Muscle strength at young age is not associated with future development of type 2 diabetes in Japanese male athletes. J Phys Fit Sports Med. 2017;6(3):167-173.

23. Mitchell JH, Haskell W, Snell P, Van Camp SP. Task Force 8: classification of sports. J Am Coll Cardiol. 2005;45(8):1364-1367.

24. Rice SG. Medical conditions affecting sports participation. Pediatrics. 2008;121(4):841-848.

25. lizuka Y, lizuka H, Mieda T, Tajika T, Yamamoto A, Takagishi K. Association between "loco-check" and EuroQol, a comprehensive instrument for assessing health-related quality of life: a study of the Japanese general population. J Orthop Sci. 2014;19(5):786-791.

26. Akahane M, Yoshihara S, Maeyashiki A, Tanaka Y, Imamura T. Lifestyle factors are significantly associated with the locomotive syndrome: a cross-sectional study. BMC Geriatrics. 2017;17(1):241.

27. Tanimoto Y, Watanabe M, Kono R, Hirota C, Takasaki K, Kono K. Aging changes in muscle mass of Japanese. Nihon Ronen Igakkai Zasshi. 2010;47(1):52-57. 
28. Kortebein P, Ferrando A, Lombeida J, Wolfe R, Evans WJ. Effect of 10 Days of Bed Rest on Skeletal Muscle in Healthy Older Adults. Jama. 2007;297(16):1769-1774.

29. Goodpaster BH, Park SW, Harris TB, Kritchevsky SB, Nevitt M, Schwartz AV, Simonsick EM, Tylavsky FA, Visser M, Newman AB et al. The Loss of Skeletal Muscle Strength, Mass, and Quality in Older Adults: The Health, Aging and Body Composition Study. The Journals of Gerontology: Series A. 2006;61(10):1059-1064.

30. Lemez S, Baker J. Do Elite Athletes Live Longer? A Systematic Review of Mortality and Longevity in Elite Athletes. Sports Med Open. 2015;1(1):16-16.

31. Sarna S, Sahi T, Koskenvuo M, Kaprio J. Increased life expectancy of world class male athletes. Med Sci Sports Exerc. 1993;25(2):237-244.

32. Teramoto M, Bungum TJ. Mortality and longevity of elite athletes. Journal of Science and Medicine in Sport. 2010;13(4):410-416.

33. Backx FJG, Beijer HJM, Bol E, Erich WBM. Injuries in high-risk persons and high-risk sports:A longitudinal study of 1818 school children. The American Journal of Sports Medicine. 1991;19(2):124-130.

34. Hootman JM, Dick R, Agel J. Epidemiology of collegiate injuries for 15 sports: summary and recommendations for injury prevention initiatives. J Athl Train. 2007;42(2):311-319.

\section{Tables}

Due to technical limitations, table 1,2,3,4 is only available as a download in the Supplemental Files section.

\section{Figures}




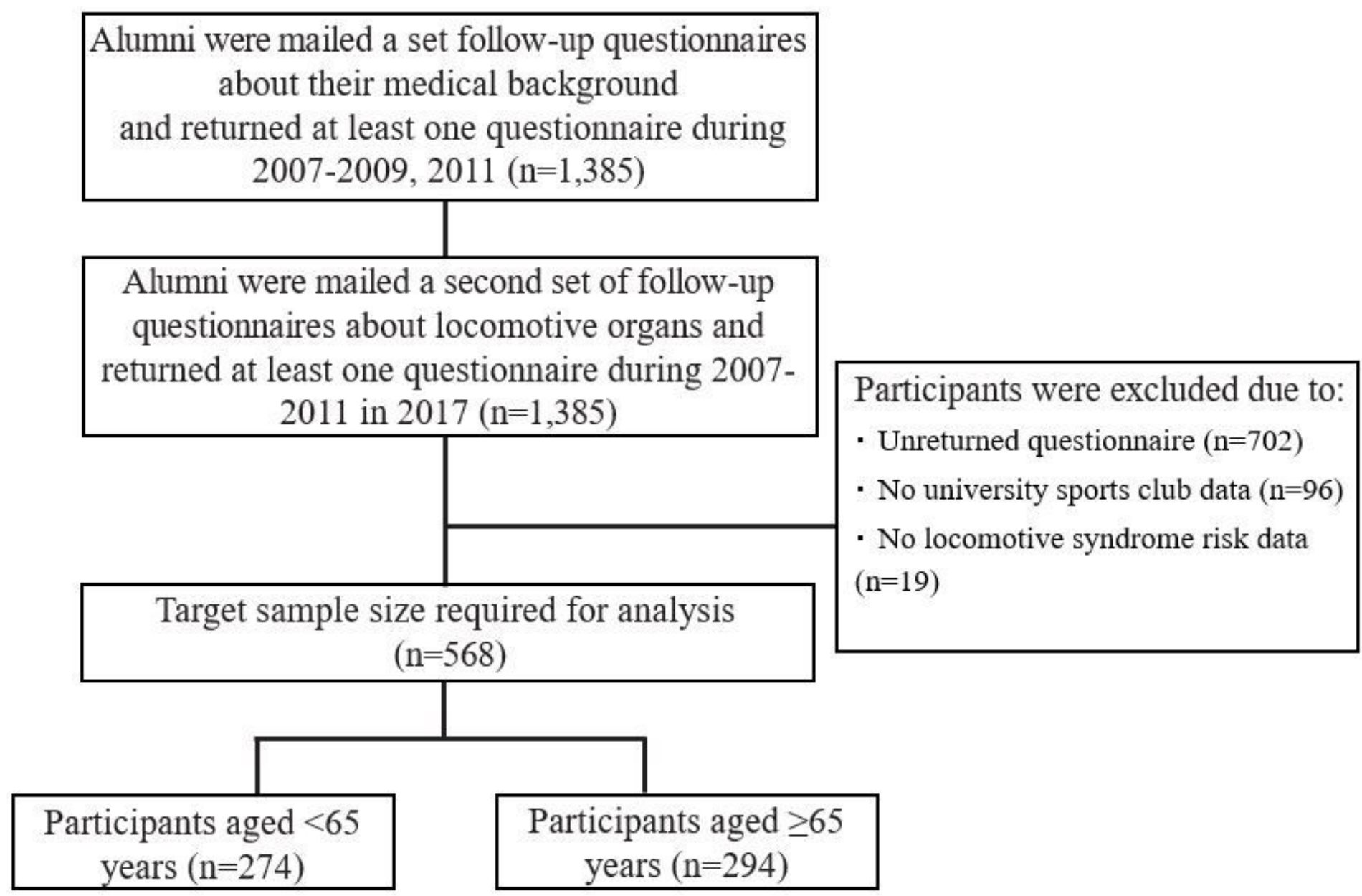

Figure 1

A flow-chart of participant inclusion steps in the present study 


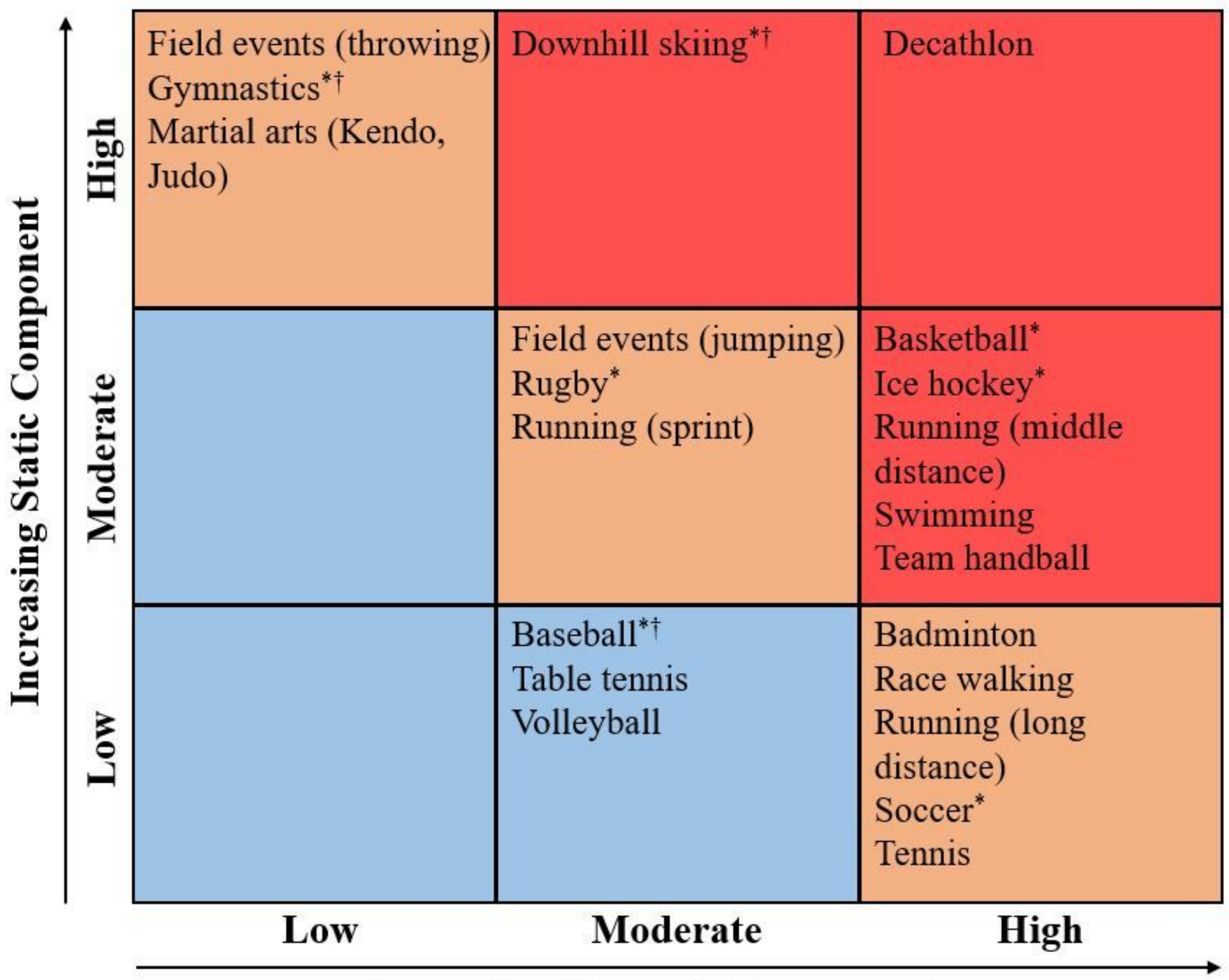

Increasing Dynamic Component

Figure 2

University sports club membership was classified into three categories of low (green), moderate (orange), high (red) total cardiovascular intensity The static component was defined as the estimated percent of maximal voluntary contraction reached; the dynamic component was defined as the estimated percent of maximal oxygen uptake achieved. The low total cardiovascular demands are shown in green (low), the moderate in orange (moderate), and the high in red (high). ${ }^{*}$ Danger of bodily collision. + Increased risk of syncope occurs.

\section{Supplementary Files}


This is a list of supplementary files associated with this preprint. Click to download.

- Additionalfile1.docx

- Tablesver1.4.xlsx 